\title{
Analysis of an Inventory System with Ramp Type Demand Rate, Partial Shortages under Inflation and Learning
}

\author{
Jayshree $^{1}$ and S.R. Singh ${ }^{2}$ \\ ${ }^{1-2}$ Department of Mathematics, C.C.S. University, Meerut, 250004 \\ Jayashri000@gmail.com, shivrajpundir@gmail.com
}

\begin{abstract}
The main purpose of this paper is to investigate the optimal replenishment policy under the learning effect and allowable shortages within the economic order quantity (EOQ) framework. We adopt a demand function which is ramp type pattern. The assumption that the goods in inventory always preserve their physical characteristics is unethical. Therefore another important factor is deterioration, as it may yield misleading results. The unit production cost is inversely proportional to the demand rate. Hence a mathematical model has been developed in the view of above scenario, in order to determine the optimal costs for two different cases, by minimizing the present worth of total costs. Finally, numerical examples are provided to illustrate the theoretical results and a sensitive analysis of the optimal solution has been performed to showcase the effect of various parameters.
\end{abstract}

Index Terms - Ramp Type Demand, Weibull Deterioration, Unit Production Cost, Shortages, inflation, learning.

\section{INTRODUCTION}

In recent years, inventory problems for deteriorating items have been widely studied. Deteriorating is a general phenomenon for many products, in which fruits or vegetables are spoiled directly while alcohol physical depletate over time, and electronic products deteriorate rapidly as time went through a gradual of loss of potential utility, that result in the decrease of usefulness of commodities. The first attempt to describe the optimal ordering policies for such items was made by Ghare and Schrader in 1963. Covert and Philip [1973] proposed an inventory model with weibull distribution rate without considering shortages. More related articles can be referred to like Yang et al. [2011], and Singh et al. [2007], Singh C.and Singh S.R. [2011], Manna and Chaudhuri [2006, 2016] and so forth.

However, for deteriorating items it is unethical that the demand rate increases continuously during their full life cycle. Based on such realistic facts, Hill proposed an inventory model with ramp type demand rate. Mandal and Pal [1998] extended this model to allow shortages. Further, Wu [2001] extended it to have Weibull distribution deterioration and time dependent backlogging. Shouri et. al. [2009] also considered a model by introducing a general ramp type demand rate, partial backlogging and Weibull deterioration rate.

While, for some short life cycle products, the demand rate may increase up to a certain level, then reach a stabilized period, and finally decrease when the inventory level falls to zero. There are many other related

Grenze ID: 01.GIJET.2.2.523

(C) Grenze Scientific Society, 2016 
literatures about such inventory model, such as Deng et. al. [2007], Giri et. al. [2003], Singh C.and Singh S.R. [2011].

Furthermore, when shortages occur, some customers are willing to wait for backorders to be fulfilled and others whom are often fickle and increasingly less loyal would not. Therefore, the occurrence of shortages in inventory is a natural phenomenon and in practice shortages are partially backlogged and partially lost. Some related works can be found in Abad [1996], Dye et al. [2007], Wu et al. [2006], Singh et al. [2009], Goyal et al. [2013].

Apart from the above mentioned facts, "learning" as natural phenomena, are observable everywhere. Learning implies that the performance of a system engaged in a repetitive task improves with time. This improvement of the system can be observed in manufacturing companies as a reduction in the cost and/ or time of production. Singh et. al. [2013], Jayshree \& Singh [2016], Kumar et al (2013), Yadav et. al. [2012] and many others have developed inventory models to cover this phenomenon.

After the global economic crisis, developing countries have suffered from large scale inflation. However, from a financial point of view, an inventory represents a capital investment and must complete with other assets for a firm's limited capital funds .Understanding of inflation and time value of money is crucial. To get the real estimate of all costs incurred, it is logical to incorporate the net profit of inflation. The pioneer research in this area was Buzzacott [1975] and Misra [1975]. Thereafter, several interesting research papers have appeared e.g. Yang et. al. [2001], Yang [2012], Singh et. al.[2008, 2009].

This paper incorporates Weibull deterioration and ramp type demand with allowable shortages under learning phenomenon. We extend the work of Jayshree \& Jain [2016] to propose an optimal replenishment policy within the EOQ framework and also carry out a sensitivity analysis of the main parameters.

\section{ASSUMPTIONS AND NOTATIONS}

The following notations and assumptions are considered to develop the inventory model

\section{A. Notations}

K- Unit Production cost (units /unit time)

$X_{1}$-Holding cost per order is partly constant and partly decreases in each cycle due to learning effect and defined as $X_{01}+\frac{X_{1}^{\prime}}{n^{k}}, k>0$

$X_{3}$ - Deterioration cost per order is partly constant and partly decreases in each cycle due to learning effect and defined as $X_{03}+\frac{X_{3}^{\prime}}{n^{k}}, k>0$

$X_{4}$-Shortage cost per order is partly constant and partly decreases in each cycle due to learning effect and defined as $X_{04}+\frac{X_{4}^{\prime}}{n^{k}}, k>0$

$X_{5}-$ Lost sales cost per order is partly constant and partly decreases in each cycle due to learning effect and defined as $X_{05}+\frac{X_{5}^{\prime}}{n^{k}}, k>0$

$\mathrm{X}$ - Total average cost for a production cycle

r- Inflationary rate

$\delta$ - Backlogging rate

\section{B. Assumptions}

(1) Demand rate in ramp type function of time, i.e. demand rate $\mathrm{R}=\mathrm{f}(\mathrm{t})$ is assumed to be a ramp type function of time $\mathrm{f}(\mathrm{t})=\mathrm{D}_{0}[\mathrm{t}-(\mathrm{t}-\mu) \mathrm{H}(\mathrm{t}-\mu)], \mathrm{D}_{0}>0$ and $\mathrm{H}(\mathrm{t})$ is a Heaviside's function: $\mathrm{H}(\mathrm{t}-\mu)= \begin{cases}1 & \text { if } t \geq \mu \\ 0 & \text { if } t<\mu\end{cases}$

(2) Deterioration varies unit time and it is function of two parameter Weibull distribution of the time, i.e. $\alpha \beta t^{\beta-1}, 0<\alpha<1, \beta \geq 1$, where $\mathrm{t}$ denote time of deterioration .

(3) Lead time is zero.

(4) Inflation is considered.

(5) Learning phenomenon is also considered.

(6) Shortage are Allowed and partially backlogged.

(7) $\mathrm{K}=\gamma \mathrm{f}(\mathrm{t})$ is the production rate where $\gamma(>1)$ is a constant. 
The unit production $\operatorname{cost} v=\alpha_{1} R^{-s}$ where $\alpha_{1}>0, s>0$ and $\mathrm{s} \neq 2$.

$\alpha_{1}$ is obviously positive since $v$ and $\mathrm{R}$ are both non-negative. Also higher demands result in lower unit cost of production. This implies that $v$ and $\mathrm{R}$ are inversely related and hence, must be non-negative i.e. positive.

Now,

$$
\begin{gathered}
\frac{d V}{d R}=-\alpha_{1} s R^{-(s+1)}<0 . \\
\frac{d^{2} V}{d R^{2}}=\alpha_{1} s(s+1) R^{-(s+2)}>0 .
\end{gathered}
$$

Thus, marginal unit cost of production is an increasing function of $\mathrm{R}$. These results imply that, as the demand rate increases, the unit cost of production decreases at an increasing rate. Due to this reason, the manufacture is encouraged to produce more as the demand for the item increases. The necessity of restriction $\mathrm{s} \neq 2$ arises from the nature of the solution of the problem.

\section{MATHEMATICAL FORMULATION OF THE MODEL}

Case $1\left(\mu \leq t_{1} \leq \mathrm{t}_{2}\right)$

The stock level initially is zero. Production starts just after $\mathrm{t}=0$. When the stock attains a level $\mathrm{q}$ at time $\mathrm{t}=$ $t_{1}$, then the production stops at that time. The time point $\mu$ occurs before the point $\mathrm{t}=t_{1}$, where demand is stabilized after that the inventory level diminishes due to both demand and deterioration ultimately falls to zero at time $\mathrm{t}=\mathrm{t}_{2}$. After time $t_{2}$ shortages occurs at $\mathrm{t}=\mathrm{T}$, which are partially backlogged and partially lost. Then, the cycle repeats.

Let $\mathrm{Q}(\mathrm{t})$ be the inventory level of the system at any time $\mathrm{t}(0 \leq \mathrm{t} \leq \mathrm{t} 2)$. The differential equations governing the system in the interval $\left[0, t_{2}\right]$ are given by

$$
\begin{array}{ll}
\frac{d Q(t)}{d t}+\alpha \beta t^{\beta-1} Q(t)=K-F(t) & 0 \leq t \leq \mu \\
\text { with the condition } \mathrm{Q}(0)=0 & \mu \leq t \leq t_{1} \\
\frac{d Q(t)}{d t}+\alpha \beta t^{\beta-1} Q(t)=K-F(t) & \\
\text { with the condition } Q\left(t_{1}\right)=q & \mathrm{t}_{1} \leq t \leq t_{2} \\
\frac{d Q(t)}{d t}+\propto \beta t^{\beta-1} Q(t)=-F(t) & \\
\text { with the condition } Q\left(t_{1}\right)=q, Q\left(t_{2}\right)=0 & \mathrm{t}_{2} \leq t \leq \mathrm{T} \\
\frac{d Q(t)}{d t}=-e^{-\delta\left(T-\mathrm{t}_{2}\right)} F(t) &
\end{array}
$$

with the condition $Q\left(t_{2}\right)=0$

Using ramp type function $\mathrm{F}(\mathrm{t})$, equation (1),(2),(3),(4) become respectively

$$
\begin{array}{ll}
\frac{d Q(t)}{d t}+\propto \beta t^{\beta-1} Q(t)=(\gamma-1) D_{0} t & 0 \leq t \leq \mu \\
\text { with the condition } \mathrm{Q}(0)=0 & \mu \leq t \leq \mathrm{t}_{1} \\
\frac{d Q(t)}{d t}+\propto \beta t^{\beta-1} Q(t)=(\gamma-1) D_{0} \mu & \\
\text { with the condition } Q\left(t_{1}\right)=q & \mathrm{t}_{1} \leq t \leq \mathrm{t}_{2} \\
\frac{d Q(t)}{d t}+\propto \beta t^{\beta-1} Q(t)=D_{0} \mu & \\
\text { With the conditions } Q\left(t_{1}\right)=\mathrm{q}, Q\left(t_{2}\right)=0, & \mathrm{t}_{2} \leq t \leq \mathrm{T}
\end{array}
$$

with the condition $\mathrm{Q}\left(\mathrm{t}_{2}\right)=0$

(5),(6),(7),(8) are first order linear differential equations

For the solution of equation (5) we get

$Q(t) e^{\alpha t^{\beta}}=(\gamma-1) \int D_{0} t e^{\alpha t^{\beta}}+C$

$=(\gamma-1) D_{0}\left[\frac{t^{2}}{2}+\frac{\alpha t^{\beta+2}}{\beta+2}+\frac{\alpha^{2} t^{2 \beta+2}}{2(2 \beta+2)}+--\right]+C$

By using the condition $\mathrm{Q}(0)=0$

$Q(t)=(\gamma-1) D_{0} e^{-\alpha t^{\beta}}\left[\frac{t^{2}}{2}+\frac{\alpha t^{\beta+2}}{(\beta+2)}+\frac{\alpha^{2} t^{2 \beta+2}}{2(2 \beta+2)}+--\right], 0 \leq t \leq \mu$

for the solution of equation (6) we have 


$$
\begin{gathered}
\int_{\mu}^{t} d\left[e^{\alpha t^{\beta}} Q(t)\right]=(\gamma-1) D_{0} \mu \int_{\mu}^{t} e^{\alpha t^{\beta}} d t \\
=(\gamma-1) D_{0} \mu e^{-\alpha t^{\beta}}\left[t-\frac{\mu}{2}+\frac{\alpha t^{\beta+1}}{(\beta+1)}+\frac{\alpha^{2} t^{2 \beta+1}}{2(2 \beta+1)}-\frac{\alpha \mu^{\beta+1}}{(\beta+1)(\beta+2)}-\frac{\alpha^{2} \mu^{2 \beta+1}}{2(2 \beta+1)(2 \beta+2)}\right], \mu \leq t \leq \mathrm{t}_{1}
\end{gathered}
$$

The solution of equation (7) is given by

$\mathrm{Q}(\mathrm{t}) e^{\alpha t^{\beta}}=-D o \mu\left(t+\frac{\alpha t^{\beta+1}}{\beta+1}+\frac{\alpha^{2} t^{2 \beta+1}}{2(2 \beta+1)}+\quad\right)+C$

Putting $\mathrm{Q}(\mathrm{t})_{1}=\mathrm{q}$ we get

$\mathrm{C}=\mathrm{q} e^{\alpha t_{1}^{\beta}}+\operatorname{Do} \mu\left(t_{1}+\frac{\alpha t_{1} \beta+1}{\beta+1}+\frac{\alpha^{2} t_{1}^{2 \beta+1}}{2(2 \beta+1)}+\quad\right)$

Using initial condition $\mathrm{Q}\left(\mathrm{t}_{2}\right)=0$ in equation (13) we have,

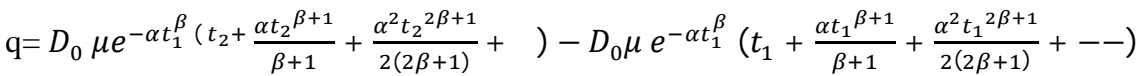

Substitute q in equation (13) the solution of equation (7) is

$Q(t)=D_{0} \mu e^{-\alpha t^{\beta}}\left[\left(t_{2}-t\right)+\frac{\alpha}{\beta+1}\left(t_{2}{ }^{\beta+1}-t^{\beta+1}\right)+\frac{\alpha^{2}}{2(2 \beta+1)}\left(t_{2}{ }^{2 \beta+1}-t^{2 \beta+1}\right)+--\right]$
$t_{1} \leq t \leq t_{2}$

The solution of equation (8) is

$$
t_{1} \leq t \leq t_{2}
$$

$\frac{d Q(t)}{d t}=-D_{0} \mu e^{-\delta\left(T-t_{2}\right)}$

$t_{2} \leq t \leq T$

with boundary condition $Q\left(t_{2}\right)=0$, we get

$Q(t)=D_{0} \mu\left[\left(t_{2}-t\right)-\delta\left(T-t_{2}\right)\left(t_{2}-t\right)\right]$

Shortage cost over the period $[0, \mathrm{~T}]$ is defined as

$=-D_{0} \mu\left[\left\{T t_{2}-\frac{t_{2}^{2}}{2}-\frac{T^{2}}{2}-\delta\left(\frac{3 t_{2} T^{2}}{2}-\frac{3 t_{2}^{2} T}{2}-\frac{T^{2}}{2}+\frac{t_{2}^{3}}{2}\right)-\left\{r \frac{T^{2} t_{2}}{2}+r \frac{t_{2}^{3}}{2}+r \frac{T^{3}}{3}+\delta r\left(T-t_{2}\right)\left(\frac{t_{2} T^{2}}{2}-\frac{t_{2}^{3}}{6}-\right.\right.\right.\right.$ $\left.\left.\left.\frac{T^{3}}{3}\right)\right\}\right]$ -

Lost sales cost per cycle is

$\mathrm{LS}=D_{0} \mu \int_{t_{2}}^{T}\left(1-e^{-\delta\left(T-t_{2}\right)}\right) d t$

$\mathrm{LS}=D_{0} \mu \int_{t_{2}}^{T} \delta\left(T-t_{2}\right)\left(t_{2}-t\right) d t$

Lost sale cost over the period $[0, \mathrm{~T}]$ is given by

$\mathrm{LS}=D_{0} \mu \delta\left[\frac{3 T^{2} t_{2}}{2}-\frac{3 t_{2}^{2} T}{2}-\frac{T^{3}}{2}+\frac{t_{2}^{3}}{2}-\frac{5 r t_{2} T^{3}}{6}-\frac{r t_{2}^{4}}{6}+\frac{r T t_{2}^{3}}{6}+\frac{r T^{4}}{3}+\frac{r t_{2}^{2} T^{2}}{2}\right]$

The total inventory over the period $\left[0, \mathrm{t}_{2}\right]$ is

$$
\begin{aligned}
& \int_{0}^{t_{2}} Q(t) d t e^{-r t}=\int_{0}^{\mu} Q(t) e^{-r t} d t+\int_{\mu}^{t_{1}} Q(t) e^{-r t} d t+\int_{t_{1}}^{0} Q(t) e^{-r t} d t \\
& \int_{0}^{\mu} Q(t) e^{-r t} d t=\int_{0}^{\mu}(\gamma-1) D_{0} e^{-\alpha t^{\beta}}\left[\frac{t^{2}}{2}+\frac{\alpha t^{\beta+2}}{(\beta+2)}+\frac{\alpha^{2} t^{2 \beta+2}}{2(2 \beta+2)}+--\right] e^{-r t} d t \\
& =(\gamma-1) D_{0}\left[\frac{\mu^{3}}{6}-\frac{\alpha \beta \mu^{\beta+3}}{2(\beta+2)(\beta+3)}-\frac{\alpha^{2}(3 \beta+2) \mu^{2 \beta+3}}{2(\beta+2)(2 \beta+2)(2 \beta+3)}-\frac{r \mu^{2}}{8}+\frac{r \alpha \beta \mu^{\beta+4}}{2(\beta+2)(\beta+4)}-\frac{r \alpha^{2}(3 \beta+2) \mu^{2 \beta+4}}{2(\beta+2)(2 \beta+2)(2 \beta+4)}\right] \\
& \int_{\mu}^{t_{1}} Q(t) e^{-r t} d t=D_{0} \mu(\gamma-1) \int_{\mu}^{t_{1}}\left[t-\frac{\mu}{2}+\frac{\alpha t^{\beta+1}}{(\beta+1)}+\frac{\alpha^{2} t^{2 \beta+1}}{2(2 \beta+1)}-\frac{\alpha \mu^{\beta+1}}{(\beta+1)(\beta+2)}-\frac{\alpha^{2} \mu^{2 \beta+1}}{2(2 \beta+1)(2 \beta+2)}-\alpha t^{\beta+1}+\frac{\alpha \mu t^{\beta}}{2}-\right. \\
& \left.\frac{\alpha^{2} t^{2 \beta+1}}{(\beta+1)}+\frac{\alpha^{2} t^{\beta} \mu^{\beta+1}}{(\beta+1)(\beta+2)}\right] e^{-r t} d t \\
& =D_{0} \mu(\gamma-1) \int_{\mu}^{t_{1}} t-\frac{\mu}{2}+\frac{\alpha t^{\beta+1}}{(\beta+1)}+\frac{\alpha^{2} t^{2 \beta+1}}{2(2 \beta+1)}-\frac{\alpha \mu^{\beta+1}}{(\beta+1)(\beta+2)}-\frac{\alpha^{2} \mu^{2 \beta+1}}{2(2 \beta+1)(2 \beta+2)}-\alpha t^{\beta+1}+\frac{\alpha \mu t^{\beta}}{2}-\frac{\alpha^{2} t^{2 \beta+1}}{(\beta+1)}+ \\
& \frac{\alpha^{2} t^{\beta} \mu^{\beta+1}}{(\beta+1)(\beta+2)}-r t^{2}+r t \frac{\mu}{2}-\frac{\alpha r t^{\beta+2}}{(\beta+1)}-\frac{r \alpha^{2} t^{2 \beta+2}}{2(2 \beta+1)}+\frac{\alpha r \mu^{\beta+1} t}{(\beta+1)(\beta+2)}+\alpha t^{\beta+2} r-\frac{r \alpha \mu t^{\beta+1}}{2}+\frac{r \alpha^{2} t^{2 \beta+2}}{(\beta+1)}-\frac{r \alpha^{2} t^{\beta+1} \mu^{\beta+1}}{(\beta+1)(\beta+2)} \\
& \int_{t_{1}}^{t_{2}} Q(t) e^{-r t} d t=D_{0} \mu \int_{t_{1}}^{t_{2}}\left[\left(t_{2}-t\right)+\frac{\alpha}{(\beta+1)}\left(t_{2}{ }^{\beta+1}-t^{\beta+1}\right)+\frac{\alpha^{2}}{2(2 \beta+1)}\left(t_{2}{ }^{2 \beta+1}\right.\right. \\
& \left.\left.\quad-t^{2 \beta+1}\right)\right] e^{-\alpha t^{\beta}} e^{-r t} d t
\end{aligned}
$$


$=D_{0} \mu\left[\frac{t_{2}^{2}}{2}-t_{2} t_{1}+\frac{t_{1}^{2}}{2}+\frac{(\alpha) t_{2}^{\beta+2} \beta}{(\beta+1)(\beta+2)}-\frac{\alpha t_{1} t_{2}^{\beta+1}}{(\beta+1)}-\frac{\alpha \beta t_{1}^{\beta+2}}{(\beta+1)(\beta+2)}-\frac{\alpha^{2} t_{2}^{2 \beta+1} t_{1}}{2(2 \beta+1)}+\frac{\alpha^{2} t_{2}^{2 \beta+2}(\beta+3)}{2(2 \beta+2)(\beta+1)}+\frac{\alpha^{2} t_{1}^{2 \beta+2}(5 \beta+3)}{2(2 \beta+1)(2 \beta+2)(\beta+1)}+\right.$
$\frac{\alpha t_{2} t_{1}^{\beta+1}}{(\beta+1)}-\frac{\alpha^{2} t_{2}^{\beta+1} t_{1}^{\beta+1}}{(\beta+1)^{2}}-\frac{r t_{2}^{2}}{2}+r t_{2} t_{1}-\frac{r t_{1}^{2}}{2}-\frac{r \alpha t_{2}^{\beta+2}}{(\beta+1)}+\frac{r \alpha t_{2}^{\beta+1} t_{1}}{(\beta+1)}+\frac{r \alpha t_{1}^{\beta+3} \beta}{(\beta+3)(\beta+1)}-\frac{\alpha^{2} t_{2}^{2 \beta+2} r}{2(2 \beta+1)}+\frac{r \alpha t_{2}^{\beta+3}(2 \beta+3)}{(\beta+1)(\beta+2)(\beta+3)}-$
$\left.\frac{3 \alpha^{2} \beta t_{2}^{2 \beta+3} r}{2(2 \beta+1)(\beta+2)(2 \beta+3)}-\frac{\alpha^{2}(5 \beta+3) t_{1}^{2 \beta+3} r}{2(2 \beta+1)(\beta+1)(2 \beta+3)}-\frac{\alpha t_{2} t_{1}^{\beta+2}}{(\beta+2)}+\frac{\alpha^{2} t_{2}^{\beta+1} t_{1}^{\beta+2}}{(\beta+1)(\beta+2)}\right]$

Therefore, the total inventory in $\left[0, \mathrm{t}_{2}\right]$ is given by

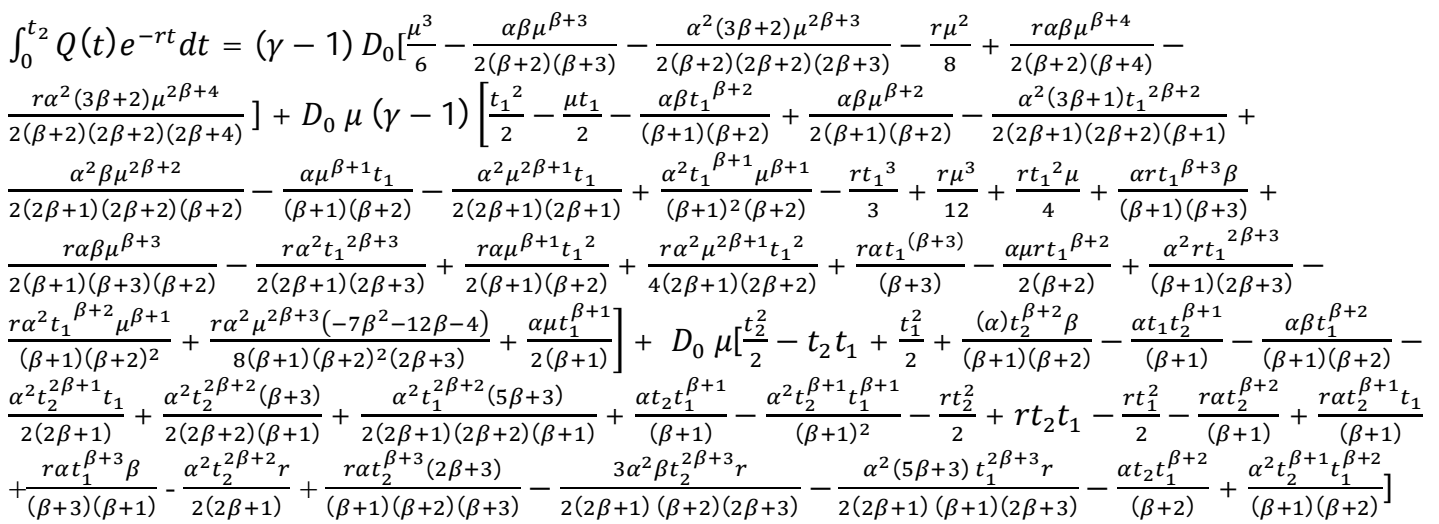

Total number of deteriorated items over the period $\left[0, \mathrm{t}_{2}\right]$ is given by

Production in $[0, \mu]+$ Production in $\left[\mu \mathrm{t}_{1}\right]-$ Demand in $[0, \mu]-$ Demand in $\left[\mu, \mathrm{t}_{2}\right]$

$=\gamma \int_{o}^{\mu} D o t e^{-r t} d t+\gamma \int_{o}^{t_{1}} D_{0} \mu e^{-r t} d t-D_{0} \int_{o}^{\mu} t e^{-r t} d t-\int_{\mu}^{t_{2}} D o \mu e^{-r t} d t$

$=\frac{1}{2} \gamma D_{0} \mu\left[2 t_{1}-\mu-r t_{1}^{2}+\frac{r \mu^{2}}{3}\right]-\frac{1}{2} D_{0} \mu\left[2 t_{2}-\mu-r t_{2}^{2}+\frac{r \mu^{2}}{3}\right]$

The cost of production in $[u, u+d u]$ is

$\mathrm{Kv} \mathrm{d} u=\frac{\alpha_{1} \gamma}{R^{s-1}}$

Hence the production cost over the period $\left[0, t_{1}\right]$ is given by

$\int_{o}^{t_{1}} \mathrm{Kv} e^{-r u} d u=\int_{0}^{\mu} \mathrm{Kv} e^{-r u} d u+\int_{\mu}^{t_{1}} \mathrm{Kv} e^{-r u} d u$

$=\int_{o}^{\mu} \frac{\alpha_{1} \gamma}{R^{s-1}} e^{-r u} d u+\int_{\mu}^{t_{1}} \frac{\alpha_{1} \gamma}{R^{s-1}} e^{-r u} d u$

$=\alpha_{1} \gamma D_{0}^{1-s}\left[\frac{(s-1) \mu^{2-s}+(2-s) \mu^{1-s} t_{1}}{(2-s)}\right]+\alpha_{1} \gamma r D_{0}{ }^{1-s}\left[\frac{\mu}{2}^{3-s}-{\frac{t_{1}}{2}}^{2} \mu^{1-s}-\frac{\mu^{1-s}}{(1-s)}\right]$

The total average inventory cost $\mathrm{X}$ is given by

$\mathrm{X}=$ Inventory Cost + Deterioration Cost + Production Cost + Shortage Cost + Lost Sales Cost

$$
\begin{aligned}
& \mathrm{X}=\frac{1}{t_{2}}\left[X _ { 1 } \left\{(\gamma-1) D_{0}\left(\frac{\mu^{3}}{6}-\frac{\alpha \beta \mu^{\beta+3}}{2(\beta+2)(\beta+3)}-\frac{\alpha^{2}(3 \beta+2) \mu^{2 \beta+3}}{2(\beta+2)(2 \beta+2)(2 \beta+3)}-\frac{r \mu^{2}}{8}+\frac{r \alpha \beta \mu^{\beta+4}}{2(\beta+2)(\beta+4)}-\frac{r \alpha^{2}(3 \beta+2) \mu^{2 \beta+4}}{2(\beta+2)(2 \beta+2)(2 \beta+4)}\right)+D_{0}\right.\right. \\
& \mu(\gamma-1)\left(\frac{t_{1}{ }^{2}}{2}-\frac{\mu t_{1}}{2}-\frac{\alpha \beta t_{1}{ }^{\beta+2}}{(\beta+1)(\beta+2)}+\frac{\alpha \beta \mu^{\beta+2}}{2(\beta+1)(\beta+2)}-\frac{\alpha^{2}(3 \beta+1) t_{1}{ }^{2 \beta+2}}{2(2 \beta+1)(2 \beta+2)(\beta+1)}+\frac{\alpha^{2} \beta \mu^{2 \beta+2}}{2(2 \beta+1)(2 \beta+2)(\beta+2)}-\frac{\alpha \mu^{\beta+1} t_{1}}{(\beta+1)(\beta+2)}-\right. \\
& \frac{\alpha^{2} \mu^{2 \beta+1} t_{1}}{2(2 \beta+1)(2 \beta+2)}+\frac{\alpha^{2} t_{1}^{\beta+1} \mu^{\beta+1}}{(\beta+1)^{2}(\beta+2)}-\frac{r t_{1}^{3}}{3}+\frac{r \mu^{3}}{12}+\frac{r t_{1}^{2} \mu}{4}+\frac{\alpha r t_{1} \beta+3}{(\beta+1)(\beta+3)}+\frac{r \alpha \beta \mu^{\beta+3}}{2(\beta+1)(\beta+3)(\beta+2)}-\frac{r \alpha^{2} t_{1}^{2 \beta+3}}{2(2 \beta+1)(2 \beta+3)}+ \\
& \frac{r \alpha \mu^{\beta+1} t_{1}{ }^{2}}{2(\beta+1)(\beta+2)}+\frac{r \alpha^{2} \mu^{2 \beta+1} t_{1}{ }^{2}}{4(2 \beta+1)(2 \beta+2)}+\frac{r \alpha t_{1}(\beta+3)}{(\beta+3)}-\frac{\alpha \mu r t_{1}^{\beta+2}}{2(\beta+2)}+\frac{\alpha^{2} r t_{1}{ }^{2 \beta+3}}{(\beta+1)(2 \beta+3)}-\frac{r \alpha^{2} t_{1}{ }^{\beta+2} \mu^{\beta+1}}{(\beta+1)(\beta+2)^{2}}+\frac{r \alpha^{2} \mu^{2 \beta+3}\left(-7 \beta^{2}-12 \beta-4\right)}{8(\beta+1)(\beta+2)^{2}(2 \beta+3)}+ \\
& \left.\frac{\alpha \mu t_{1}^{\beta+1}}{2(\beta+1)}\right)+D_{0} \mu\left(\frac{t_{2}^{2}}{2}-t_{2} t_{1}+\frac{t_{1}^{2}}{2}+\frac{(\alpha) t_{2}^{\beta+2} \beta}{(\beta+1)(\beta+2)}-\frac{\alpha t_{1} t_{2}^{\beta+1}}{(\beta+1)}-\frac{\alpha \beta t_{1}^{\beta+2}}{(\beta+1)(\beta+2)}-\frac{\alpha^{2} t_{2}^{2 \beta+1} t_{1}}{2(2 \beta+1)}+\frac{\alpha^{2} t_{2}^{2 \beta+2}(\beta+3)}{2(2 \beta+2)(\beta+1)}+\right.
\end{aligned}
$$


$\frac{\alpha^{2} t_{1}^{2 \beta+2}(5 \beta+3)}{2(2 \beta+1)(2 \beta+2)(\beta+1)}+\frac{\alpha t_{2} t_{1}^{\beta+1}}{(\beta+1)}-\frac{\alpha^{2} t_{2}^{\beta+1} t_{1}^{\beta+1}}{(\beta+1)^{2}}-\frac{r t_{2}^{2}}{2}+r t_{2} t_{1}-\frac{r t_{1}^{2}}{2}-\frac{r \alpha t_{2}^{\beta+2}}{(\beta+1)}+\frac{r \alpha t_{2}^{\beta+1} t_{1}}{(\beta+1)}+\frac{r \alpha t_{1}^{\beta+3} \beta}{(\beta+3)(\beta+1)}-\frac{\alpha^{2} t_{2}^{2 \beta+2} r}{2(2 \beta+1)}-$ $\left.\left.\frac{r \alpha t_{2}^{\beta+3}(2 \beta+3)}{(\beta+1)(\beta+2)(\beta+3)}-\frac{3 \alpha^{2} \beta t_{2}^{2 \beta+3} r}{2(2 \beta+1)(\beta+2)(2 \beta+3)}-\frac{\alpha^{2}(5 \beta+3) t_{1}^{2 \beta+3} r}{2(2 \beta+1)(\beta+1)(2 \beta+3)}-\frac{\alpha t_{2} t_{1}^{\beta+2}}{(\beta+2)}+\frac{\alpha^{2} t_{2}^{\beta+1} t_{1}^{\beta+2}}{(\beta+1)(\beta+2)}\right)\right\}+X_{3}\left\{\frac{1}{2} \gamma D_{0} \mu\left(2 t_{1}-\mu-\right.\right.$ $\left.\left.r t_{1}^{2}+\frac{r \mu^{2}}{3}\right)-\frac{1}{2} D_{0} \mu\left(2 t_{2}-\mu-r t_{2}^{2}+\frac{r \mu^{2}}{3}\right)\right\}-X_{4} D_{0} \mu\left\{T t_{2}-\frac{t_{2}^{2}}{2}-\frac{T^{2}}{2}-\delta\left(\frac{3 t_{2} T^{2}}{2}-\frac{3 t_{2}^{2} T}{2}-\frac{T^{3}}{2}+\frac{t_{2}^{3}}{2}\right)-r \frac{T^{2} t_{2}}{2}+\right.$ $\left.r \frac{t_{2}^{3}}{2}+r \frac{T^{3}}{3}+\delta r\left(T-t_{2}\right)\left(\frac{t_{2} T^{2}}{2}-\frac{t_{2}^{3}}{6}-\frac{T^{3}}{3}\right)\right\}+X_{5} D_{0} \mu \delta\left(\frac{3 T^{2} t_{2}}{2}-\frac{3 t_{2}^{2} T}{2}-\frac{T^{3}}{2}+\frac{t_{2}^{3}}{2}-\frac{5 r t_{2} T^{3}}{6}-\frac{r t_{2}^{4}}{6}+\frac{r T t_{2}^{3}}{6}+\frac{r T^{4}}{3}+\right.$ $\left.\left.\frac{r t_{2}^{2} T^{2}}{2}\right)+\alpha_{1} \gamma D_{0}^{1-s}\left\{\frac{(s-1) \mu^{2-s}+(2-s) \mu^{1-s} t_{1}}{(2-s)}\right\}+\alpha_{1} \gamma r D_{0}^{1-s}\left\{\frac{\mu}{2}^{3-s}-{\frac{t_{1}}{2}}^{2} \mu^{1-s}-\frac{\mu^{1-s}}{(1-s)}\right\}\right](23)$

$$
X_{1}=X_{01}+\frac{\mathrm{x}_{3}^{\prime}}{\mathrm{n}^{\mathrm{k}}}
$$

Where $X_{1}$ is continuously deceases over $n$ since $\frac{\mathrm{dx}_{1}}{\mathrm{dn}}<0, \mathrm{n}>0$

$$
\mathrm{X}_{3}=\mathrm{X}_{03}+\frac{\mathrm{x}_{3}^{\prime}}{\mathrm{n}^{\mathrm{k}}}
$$

Where $X_{3}$ is continuously deceases over $n$ since $\frac{d X_{3}}{d n}<0, n>0$

$$
\mathrm{X}_{4}=X_{04}+\frac{X_{4}^{\prime}}{n^{k}}
$$

Where $X_{4}$ is continuously deceases over $\mathrm{n}$ since $\frac{\mathrm{dx}_{4}}{\mathrm{dn}}<0, \mathrm{n}>0$

$$
\mathrm{X}_{5}=\mathrm{X}_{05}+\frac{\mathrm{X}_{5}^{\prime}}{\mathrm{n}^{\mathrm{k}}}
$$

Where $X_{5}$ is continuously deceases over $n$ since $\frac{\mathrm{dX}_{4}}{\mathrm{dn}}<0, \mathrm{n}>0$

Optimum values of $t_{1}$ and $t_{2}$ for minimum average cost $X$ are the solutions of the equations

$$
\frac{\partial X}{\partial t_{1}}=0 \text { and } \frac{\partial X}{\partial t_{2}}=0
$$

Provided they satisfy the sufficient conditions

$$
\begin{gathered}
\frac{\partial^{2} X}{\partial t_{1}^{2}}>0, \frac{\partial^{2} X}{\partial t_{2}^{2}}>0 \text { and } \frac{\partial^{2} X}{\partial t_{1}^{2}} \frac{\partial^{2} X}{\partial t_{2}^{2}}-\left(\frac{\partial^{2} X}{\partial t_{1} \partial t_{2}}\right)^{2}>0 \\
\frac{\partial X}{\partial t_{1}}=0 \text { and } \frac{\partial X}{\partial t_{2}}=0 \text { gives } \\
C_{1}\left\{\begin{array}{c}
D_{0} \mu(\gamma-1)\left(t_{1}-\frac{\alpha \beta t_{1}^{\beta+1}}{(\beta+1)}-\frac{\alpha^{2}(3 \beta+1) t_{1}^{2 \beta+1}}{2(2 \beta+1)(\beta+1)}-\frac{\alpha \mu^{\beta+1}}{(\beta+1)(\beta+2)}-\frac{\alpha^{2} \mu^{2 \beta+1}}{2(2 \beta+1)(2 \beta+2)}-\frac{\mu}{2}+\frac{\alpha^{2} t_{1}^{\beta} \mu^{\beta+1}}{(\beta+1)(\beta+2)}-r t_{1}^{2}+\frac{r t_{1} \mu}{2}+\right. \\
\left.\frac{\alpha r t_{1}^{\beta+2} \beta}{(\beta+1)}-\frac{r \alpha^{2} t_{1}^{2 \beta+2}}{2(2 \beta+1)}+\frac{r \alpha \mu^{\beta+1} t_{1}}{(\beta+1)(\beta+2)}+\frac{r \alpha^{2} \mu^{2 \beta+1} t_{1}}{2(2 \beta+1)(2 \beta+2)}+r \alpha t_{1}^{\beta+2}-\frac{\alpha \mu r t_{1}^{\beta+1}}{2}+\frac{\alpha^{2} r t_{1}^{2 \beta+2}}{(\beta+1)}-\frac{r \alpha^{2} t_{1}^{\beta+1} \mu^{\beta+1}}{(\beta+1)(\beta+2)}+\frac{\alpha \mu t_{1}^{\beta}}{2}\right)+ \\
D_{0} \mu\left(-t_{2}+t_{1}-\frac{\alpha t_{2}^{\beta+1}}{(\beta+1)}-\frac{\alpha \beta t_{1}^{\beta+1}}{(\beta+1)}-\frac{\alpha^{2} t_{2}^{2 \beta+1}}{2(2 \beta+1)}+\frac{\alpha^{2} t_{1}^{2 \beta+1}(5 \beta+3)}{2(2 \beta+1)(\beta+1)}+\alpha t_{2} t_{1}^{\beta}-\frac{\alpha^{2} t_{2}^{\beta+1} t_{1}^{\beta}}{(\beta+1)}+r t_{2}-r t_{1}+\frac{r \alpha t_{2}^{\beta+1}}{(\beta+1)}+\right. \\
\left.\left.\frac{r \alpha t_{1}^{\beta+2} \beta}{(\beta+1)}-\frac{\alpha^{2}(5 \beta+3) t_{1}^{2 \beta+2} r}{2(2 \beta+1)(\beta+1)}-\alpha t_{2} t_{1}^{\beta+1}+\frac{\alpha^{2} t_{2}^{\beta+1} t_{1}^{\beta+1}}{(\beta+1)}\right)\right\}+c_{3} \gamma D_{0} \mu\left(1-r t_{1}\right)+\alpha_{1} \gamma D_{0}^{1-s} \mu^{1-s}- \\
\alpha_{1} \gamma D_{0}^{1-s} t_{1} \mu^{1-s}=0
\end{array}\right.
\end{gathered}
$$

and

$$
\begin{aligned}
& X_{1} D_{o} \mu\left(t_{2}-t_{1}+\frac{\alpha \beta t_{2}^{\beta+1}}{(\beta+1)}-\alpha t_{1} t_{2}^{\beta}-\frac{\alpha^{2} t_{1} t_{2}^{2 \beta}}{2}+\frac{\alpha^{2}(\beta+3) t_{2}^{2 \beta+1}}{2(\beta+1)}+\frac{\alpha t_{1}^{\beta+1}}{(\beta+1)}-\frac{\alpha^{2} t_{1}^{\beta+1} t_{2}^{\beta}}{(\beta+1)}-r t_{2}+r t_{1}-\frac{r \alpha(\beta+2) t_{2}^{\beta+1}}{(\beta+1)}+\right. \\
& \left.r \alpha t_{1} t_{2}^{\beta}-\frac{r \alpha^{2}(2 \beta+2) t_{2}^{2 \beta+1}}{2(2 \beta+1)}+\frac{r \alpha(2 \beta+3) t_{2}^{\beta+2}}{(\beta+1)(\beta+2)}-\frac{3 \alpha^{2} \beta r t_{2}^{2 \beta+2}}{2(2 \beta+1)(\beta+2)}-\frac{\alpha t_{1}^{\beta+2}}{(\beta+2)}+\frac{\alpha^{2} t_{2}^{\beta} t_{1}^{\beta+2}}{(\beta+2)}\right)-X_{3} D_{o} \mu\left(1-r t_{2}\right)- \\
& X_{4} D_{o} \mu\left\{\left(T-t_{2}\right)-\delta\left(\frac{3}{2} T^{2}-3 t_{2} T+\frac{3 t_{2}^{2}}{2}\right)-\frac{r T^{2}}{2}+\frac{3 r t_{2}^{2}}{2}-\delta r t_{2} T^{2}+\frac{2}{3} \delta r t_{2}^{3}+\frac{5}{6} \delta r T^{3}-\frac{\delta r T t_{2}^{2}}{2}\right\}+ \\
& X_{5} D_{o} \mu \delta\left(\frac{3}{2} T^{2}-3 t_{2} T+\frac{3}{2} t_{2}^{2}-\frac{5 r T^{3}}{6}-\frac{2}{3} r t_{2}^{3}+\frac{1}{2} r T t_{2}^{2}+r t_{2} T^{2}\right)-X=0
\end{aligned}
$$

Case-II $\left(\mathrm{t}_{1} \leq \mu \leq \mathrm{t}_{2}\right)$

The production starts with zero stock level at $t=0$. Production begins at $t=0$ and continues up to $t=t_{1}$ and stops as soon as the stock level becomes $\mathrm{L}$ at $\mathrm{t}=\mathrm{t}_{2}$. Because of reasons of market demand and deterioration of items, the inventory level decreases till it becomes again zero at $t=t_{2}$. After time $t=t_{2}$, another important factor occurs which is shortages. After that period, the cycle repeats itself. 
Let $\mathrm{Q}(\mathrm{t})$ be the inventory level of the system at any time $\mathrm{t}\left(0 \leq \mathrm{t} \leq \mathrm{t}_{2}\right)$. The differential equations governing the system in the interval $\left[0, \mathrm{t}_{2}\right]$ are given by

$$
\frac{d Q(t)}{d t}+\alpha \beta t^{\beta-1} Q(t)=K-F(t) \quad 0 \leq \mathrm{t} \leq \mathrm{t}_{1}
$$

with the condition $\mathrm{Q}(0)=0, \mathrm{Q}\left(\mathrm{t}_{1}\right)=\mathrm{L}$

$\frac{d Q(t)}{d t}+\alpha \beta t^{\beta-1} Q(t)=-F(t)$

$$
\mathrm{t}_{1} \leq \mathrm{t} \leq \mu
$$

with the condition $\mathrm{Q}\left(\mathrm{t}_{1}\right)=\mathrm{L}$

$\frac{d Q(t)}{d t}+\alpha \beta t^{\beta-1} Q(t)=-F(t)$

$$
\mu \leq \mathrm{t} \leq \mathrm{t}_{2}
$$

with the condition $\mathrm{Q}\left(\mathrm{t}_{2}\right)=0$

$$
\frac{d Q}{d t}=-e^{-\delta(T-t)} \mathrm{F}(\mathrm{t}) \quad \mathrm{t}_{2} \leq \mathrm{t} \leq \mathrm{T}
$$

with the condition $\mathrm{Q}\left(\mathrm{t}_{2}\right)=0$

using ramp type function $\mathrm{F}(\mathrm{t})$ equations $(26),(27)(28),(29)$ become respectively

$\frac{d Q(t)}{d t}+\alpha \beta t^{\beta-1} Q(t)=(\gamma-1) D_{0} t$

$0 \leq \mathrm{t} \leq \mathrm{t}_{1}$

with the condition $\mathrm{Q}(0)=0, \mathrm{Q}\left(\mathrm{t}_{1}\right)=\mathrm{L}$

$\frac{d Q(t)}{d t}+\alpha \beta t^{\beta-1} Q(t)=-D_{0} t$

$\mathrm{t}_{1} \leq \mathrm{t} \leq \mu$

with the condition $\mathrm{Q}\left(\mathrm{t}_{1}\right)=\mathrm{L}$

$\frac{\mathrm{dQ}(\mathrm{t})}{\mathrm{dt}}+\alpha \beta \mathrm{t}^{\beta-1} \mathrm{Q}(\mathrm{t})=-\mathrm{D}_{0} \mu \quad \mu \leq \mathrm{t} \leq \mathrm{t}_{2}$

with the condition $\mathrm{Q}\left(\mathrm{t}_{2}\right)=0$

$\frac{d Q}{d t}=-e^{-\delta(T-t)} \quad D_{0} \mu \quad \mathrm{t}_{2} \leq \mathrm{t} \leq \mathrm{T}$

with the condition $\mathrm{Q}\left(\mathrm{t}_{2}\right)=0$

The solution of equation (30) is given by the expression (11) and we have

$e^{\alpha t^{\beta}} Q(t)=(\gamma-1) D_{0}\left(\frac{t^{2}}{2}+\frac{\alpha t^{\beta+2}}{\beta+2}+\alpha^{2} \frac{t^{2^{\beta+2}}}{2(2 \beta+2)}+\right)+C$

With the condition $\mathrm{Q}(0)=0$, we get

$Q(t)=(\gamma-1) D_{0} e^{-\alpha t^{\beta}}\left(\frac{t^{2}}{2}+\frac{\alpha t^{\beta+2}}{\beta+2}+\frac{\alpha^{2} t^{2 \beta+2}}{2(2 \beta+2)}+\right) \quad 0 \leq \mathrm{t} \leq \mathrm{t}_{1}$

Using boundary condition $\mathrm{Q}\left(\mathrm{t}_{1}\right)=\mathrm{L}$ in (34) we get

$L=(\gamma-1) D_{0} e^{-\alpha t_{1}^{\beta}}\left(\frac{t_{1}^{2}}{2}+\frac{\alpha t_{1}^{\beta+2}}{\beta+2}+\frac{\alpha^{2} t_{1}^{2 \beta+2}}{2(2 \beta+2)}+\right)$

Therefore the solution of equation (31) is given by

$$
\begin{array}{rl}
\frac{d Q(t)}{d t}+\alpha \beta t^{\beta-1} & Q(t)=-D_{0} t \\
= & -D_{0}\left(\frac{t^{2}}{2}+\frac{\alpha t^{\beta+2}}{\beta+2}+\frac{\alpha^{2} t^{2 \beta+2}}{2(2 \beta+2)}+\right)+C
\end{array}
$$

Using condition $\mathrm{Q}\left(\mathrm{t}_{1}\right)=\mathrm{L}$

$C=L e^{\alpha t_{1}{ }^{\beta}}+D_{0}\left(\frac{t_{1}^{2}}{2}+\frac{\alpha t_{1}^{\beta+2}}{\beta+2}+\frac{\alpha^{2} t_{1}^{2 \beta+2}}{2(2 \beta+2)}+\right)$ 


$$
Q(t)=-D_{0} e^{-\alpha^{\beta}}\left(\frac{t^{2}}{2}+\frac{2 t^{\beta+2}}{(\beta+2)}+\frac{\alpha^{2} t^{2 \beta+2}}{2(2 \beta+2)}+\right)+\gamma D_{0} e^{-\alpha^{\beta}}\left(\frac{t_{1}^{2}}{2}+\frac{\alpha t_{1}^{\beta+2}}{(\beta+2)}+\frac{\alpha^{2} t_{1}^{2 \beta+2}}{2(2 \beta+2)}+\right), \mathrm{t}_{1} \leq \mathrm{t} \leq \mu
$$

Using boundary condition $\mathrm{Q}\left(\mathrm{t}_{2}\right)=0$, the solution of equation (32) is given by

$$
\mathrm{Q}(\mathrm{t})=D_{0} \mu \boldsymbol{e}^{-\alpha^{\beta}}\left(\left(t_{2}-t\right)+\frac{\alpha}{(\beta+1)}\left(t_{2}^{\beta+1}-t^{\beta+1}\right)+\frac{\alpha^{2}}{2(2 \beta+1)}\left(t_{2}^{2 \beta+1}-t^{2 \beta+2}\right)+\right), \mu \leq \mathrm{t} \leq \mathrm{t}_{2}
$$

The solution of equation (33) is given by

$$
\frac{d Q(t)}{d t}=-D_{0} \mu e^{-\delta\left(T-t_{2}\right)} \quad t_{2} \leq t \leq T
$$

By using the boundary condition $Q\left(t_{2}\right)=0$, we get

$Q(t)=D_{0} \mu\left[\left(t_{2}-t\right)-\delta\left(T-t_{2}\right)\left(t_{2-} t\right)\right]$

Total inventory over the period $\left[0, \mathrm{t}_{2}\right]$ is

$$
\begin{aligned}
& \int_{0}^{t_{2}} Q(t) e^{-r t} d t=\int_{0}^{t_{1}} Q(t) e^{-r t} d t+\int_{t_{1}}^{\mu} Q(t) e^{-r t} d t+\int_{\mu}^{t_{2}} Q(t) e^{-r t} d t \\
& \int_{0}^{t_{1}} Q(t) e^{-r t} d t=(\gamma-1) D_{0} \int_{0}^{t_{1}} e^{-\alpha t^{\beta}}\left(\frac{t^{2}}{2}+\frac{\alpha t^{\beta+2}}{\beta+2}+\frac{\alpha^{2} t^{2 \beta+2}}{2(2 \beta+2)}+\right) e^{-r t} d t \\
& =(\gamma-1) D_{0}\left[\frac{t_{1}^{3}}{6}-\frac{\alpha \beta t_{1}^{\beta+3}}{2(\beta+2)(\beta+3)}-\frac{\alpha^{2}(3 \beta+2) t_{1}^{2 \beta+3}}{2(2 \beta+2)(\beta+2)(2 \beta+3)}-\frac{r t_{1}^{4}}{8}+\frac{\alpha \beta r r_{1}^{\beta+4}}{2(\beta+2)(\beta+4)}+\frac{r \alpha^{2}(3 \beta+2) t_{1}^{2 \beta+4}}{2(2 \beta+2)(\beta+2)(2 \beta+4)}\right] \\
& \int_{t_{1}}^{\mu} Q(t) e^{-r t} d t=D_{0} \int_{t_{1}}^{\mu} e^{-\alpha t^{\beta}}\left(\frac{\gamma t_{1}^{2}}{2}+\frac{\alpha \gamma t_{1}^{\beta+2}}{(\beta+2)}+\frac{\gamma \alpha^{2} t_{1}^{2 \beta+2}}{2(2 \beta+2)}-\frac{t^{2}}{2}-\frac{\alpha t^{\beta+2}}{(\beta+2)}-\frac{\alpha^{2} t^{2 \beta+2}}{2(2 \beta+2)}\right)(1-r t)
\end{aligned}
$$

$=\mathrm{D}_{0}$

$\left[\frac{\gamma \mu t_{1}^{2}}{2}-\frac{\gamma t_{1}^{3}}{2}+\frac{\alpha \mu \gamma t_{1}^{\beta+2}}{(\beta+2)}-\frac{\alpha \gamma \beta t_{1}^{\beta+3}}{2(\beta s s+2)(\beta+1)}+\frac{\alpha^{2} \gamma t_{1}^{2 \beta+2} \mu}{2(2 \beta+2)}-\frac{\alpha \beta t_{1}^{\beta+3}}{2(\beta+2)(\beta+3)}-\frac{\mu^{3}}{6}+\frac{t_{1}^{3}}{6}+\frac{\alpha \beta \mu^{\beta+3}}{2(\beta+2)(\beta+3)}+\frac{\alpha^{2}(3 \beta+2) \mu^{2 \beta+3}}{2(2 \beta+2)(2 \beta+3)(\beta+2)}-\right.$ $\frac{\alpha \gamma \mu^{\beta+1} t_{1}^{2}}{2(\beta+1)}-\frac{\alpha^{2} \gamma \mu^{\beta+1} t_{1}^{\beta+2}}{(\beta+2)(\beta+2)}-\frac{\alpha^{2}(3 \beta+2) t_{1}^{2 \beta+3}}{2(2 \beta+2)(2 \beta+3)(\beta+2)}-\frac{\alpha^{2} \gamma(\beta-2) t_{1}^{2 \beta+3}}{2(\beta+1)(\beta+2)}-\frac{r \gamma \mu^{2} t_{1}^{2}}{4}+\frac{r \gamma t_{1}^{4}}{4}-\frac{r \alpha \gamma t_{1}^{\beta+2} \mu^{2}}{2(\beta+2)}-\frac{r \gamma \alpha^{2} t_{1}^{2 \beta+2} \mu^{2}}{8(\beta+1)}+$ $\frac{r \gamma \alpha^{2}\left(\beta^{2}-4 \beta-4\right) t_{1}^{2 \beta+4}}{8(\beta+1)(\beta+2)^{2}}+\frac{r \mu^{4}}{8}-\frac{r t_{1}^{4}}{8}-\frac{r \alpha \beta \mu^{\beta+4}}{2(\beta+2)(\beta+4)}+\frac{r \alpha \beta t_{1}^{\beta+4}}{2(\beta+2)(\beta+4)}-\frac{r \alpha^{2}(3 \beta+2) \mu^{2} \beta+4}{8(\beta+1)(\beta+2)^{2}}+\frac{r \alpha^{2}(3 \beta+2) t_{1}^{2 \beta+4}}{8(\beta+2)^{2}(\beta+1)}+\frac{r \alpha \gamma \mu^{\beta+2} t_{1}^{2}}{2(\beta+2)}+$ $\left.\frac{r \alpha^{2} \gamma t_{1}^{\beta+2} \mu^{\beta+2}}{(\beta+2)^{2}}\right]$

$\int_{\mu}^{t_{2}} Q(t) e^{-r t} d t=D_{0} \mu \int_{\mu}^{t_{2}} e^{-\alpha t^{\beta}}\left[\left(t_{2}-t_{1}\right) \frac{\alpha}{(\beta+1)}\left(t_{2}^{\beta+1}-t^{\beta+1}\right)+\frac{\alpha^{2}}{2(2 \beta+1)}\left(t_{2}^{2 \beta+1}-t^{2 \beta+1}\right)\right] e^{-r t} d t$

$=D_{0} \mu\left[\frac{t_{2}^{2}}{2}-t_{2} \mu+\frac{\mu^{2}}{2}\right)-\frac{\alpha \mu t_{2}^{\beta+1}}{(\beta+1)}+\frac{\alpha \beta t_{2}^{\beta+2}}{(\beta+1)(\beta+2)}-\frac{\alpha \beta \mu^{\beta+2}}{(\beta+1)(\beta+2)}+\frac{\alpha^{2}(\beta-1) t_{2}^{2 \beta+2}}{2(2 \beta+2)(\beta+1)}-\frac{\alpha^{2} \mu t_{2}^{2 \beta+1}}{2(2 \beta+1)}-\frac{\alpha^{2}(3 \beta+1) \mu^{2 \beta+2}}{2(2 \beta+1)(2 \beta+2)(\beta+1)}+$ $\frac{\alpha^{2} \mu^{\beta+1} t_{2}^{\beta+1}}{(\beta+1)^{2}}+\frac{\alpha \mu^{\beta+1} t_{2}}{(\beta+1)}-\frac{r t_{2}^{3}}{6}+\frac{r \mu^{2} t_{2}}{2}-\frac{r \mu^{3}}{3}-\frac{\alpha \beta r t_{2}^{\beta+3}}{2(\beta+2)(\beta+3)}+\frac{r \alpha \mu^{2} t_{2}^{\beta+1}}{2(\beta+1)}+\frac{\alpha r \beta \mu^{\beta+3}}{(\beta+1)(\beta+3)}-\frac{r \alpha \mu^{\beta+2} t_{2}}{(\beta+2)}-\frac{r \alpha^{2}(\beta-2) t_{2}^{2 \beta+3}}{4(\beta+2)(2 \beta+3)}+$ $\left.\frac{r \alpha^{2} \mu^{2} t_{2}^{2 \beta+1}}{4(2 \beta+1)}+\frac{r \alpha^{2}(3 \beta+1) \mu^{2 \beta+3}}{2(\beta+1)(2 \beta+3)(2 \beta+1)}\right]$

Total inventory over the period $\left[0, t_{2}\right]$ is given by

$\int_{0}^{t_{2}} Q(t) e^{-r t} d t=$

$(\gamma-1) D_{0}\left[\frac{t_{1}^{3}}{6}-\frac{\alpha \beta t_{1}^{\beta+3}}{2(\beta+2)(\beta+3)}-\frac{\alpha^{2}(3 \beta+2) t_{1}^{2 \beta+3}}{2(2 \beta+2)(\beta+2)(2 \beta+3)}-\frac{r t_{1}^{4}}{8}+\frac{\alpha \beta r t_{1}^{\beta+4}}{2(\beta+2)(\beta+4)}+\frac{r \alpha^{2}(3 \beta+2) t_{1}^{2 \beta+4}}{2(2 \beta+2)(\beta+2)(2 \beta+4)}\right]+D_{0}\left[\frac{\gamma \mu t_{1}^{2}}{2}-\frac{\gamma t_{1}^{3}}{2}+\right.$ $\frac{\alpha \gamma t_{1}^{\beta+2} \mu^{2}}{\beta+2}-\frac{\alpha \gamma \beta t_{1}^{\beta+3}}{2(\beta+2)(\beta+1)}+\frac{\alpha^{2} \gamma t_{1}^{2 \beta+2} \mu}{2(2 \beta+2)}-\frac{\alpha \beta t_{1}^{\beta+3}}{2(\beta+2)(\beta+3)}-\frac{\mu^{3}}{6}+\frac{t_{1}^{3}}{6}+\frac{\alpha \beta \mu^{\beta+3}}{2(\beta+2)(\beta+3)}+\frac{\alpha^{2}(3 \beta+2) \mu^{2 \beta+3}}{2(2 \beta+2)(2 \beta+3)(\beta+2)}-\frac{\alpha \gamma \mu^{\beta+1} t_{1}^{2}}{2(\beta+1)}-$ $\frac{\alpha^{2} \gamma \mu^{\beta+1} t_{1}^{\beta+2}}{(\beta+1)(\beta+2)}-\frac{\alpha^{2}(3 \beta+2) t_{1}^{2 \beta+3}}{2(2 \beta+2)(2 \beta+3)(\beta+2)}-\frac{\alpha^{2} \gamma(\beta-2) t_{1}^{2 \beta+3}}{4(\beta+1)(\beta+2)}-\frac{r \gamma t_{1}^{2} \mu^{2}}{4}+\frac{r \gamma t_{1}^{4}}{4}-\frac{r \alpha \gamma t_{1}^{\beta+2} \mu^{2}}{2(\beta+2)}-\frac{r \gamma \alpha^{2} t_{1}^{2 \beta+2} \mu^{2}}{8(\beta+1)}+$ 


$$
\begin{aligned}
& \frac{r \gamma \alpha^{2}\left(\beta^{2}-4 \beta-4\right) t_{1}^{2 \beta+4}}{8(\beta+1)(\beta+2)^{2}}+\frac{r \mu^{4}}{8}-\frac{r t_{1}^{4}}{8}-\frac{r \alpha \beta \mu^{\beta+4}}{2(\beta+2)(\beta+4)}+\frac{r \alpha \beta t_{1}^{\beta+4}}{2(\beta+2)(\beta+4)}-\frac{r \alpha^{2}(3 \beta+2) \mu^{2 \beta+4}}{8(\beta+1)(\beta+2)^{2}}+\frac{r \alpha^{2}(3 \beta+2) t_{1}^{2 \beta+4}}{8(\beta+2)^{2}(\beta+1)}+\frac{r \alpha \gamma \mu^{\beta+2} t_{1}^{2}}{2(\beta+2)}+ \\
& \left.\frac{r \alpha^{2} \gamma t_{1}^{\beta+2} \mu^{\beta+2}}{(\beta+2)^{2}}\right]+D_{0} \mu\left[\frac{t_{2}^{2}}{2}-t_{2} \mu+\frac{\mu^{2}}{2}\right]-\frac{\alpha \mu t_{2}^{\beta+1}}{(\beta+1)}+\frac{\alpha \beta t_{2}^{\beta+2}}{(\beta+1)(\beta+2)}-\frac{\alpha \beta \mu^{\beta+2}}{(\beta+1)(\beta+2)}+\frac{\alpha \mu^{\beta+1} t_{2}}{(\beta+1)}+\frac{\alpha^{2}(\beta-1) t_{2}^{2 \beta+2}}{2(2 \beta+2)(\beta+1)}- \\
& \frac{\alpha^{2} \mu t_{2}{ }^{2 \beta+1}}{2(2 \beta+1)}-\frac{\alpha^{2}(3 \beta+1) \mu^{2 \beta+2}}{2(2 \beta+1)(2 \beta+2)(\beta+1)}+\frac{\alpha^{2} \mu^{\beta+1} t_{2} \beta+1}{(\beta+1)^{2}}-\frac{r t_{2}^{3}}{6}+\frac{r \mu^{2} t_{2}}{2}-\frac{r \mu^{3}}{3}-\frac{\alpha \beta r t_{2}^{\beta+3}}{2(\beta+2)(\beta+3)}+\frac{r \alpha \mu^{2} t_{2}^{\beta+1}}{2(\beta+1)}+\frac{\alpha r \beta \mu^{\beta+3}}{(\beta+1)(\beta+3)}- \\
& \left.\frac{r \alpha \mu^{\beta+2} t_{2}}{(\beta+2)}-\frac{r \alpha^{2}(\beta-2) t_{2}^{2 \beta+3}}{4(\beta+2)(2 \beta+3)}+\frac{r \alpha^{2} \mu^{2} t_{2}^{2 \beta+1}}{4(2 \beta+1)}+\frac{r \alpha^{2}(3 \beta+1) \mu^{2 \beta+3}}{2(\beta+1)(2 \beta+3)(2 \beta+1)}\right]
\end{aligned}
$$

The number of deteriorated items over the period $\left[0, t_{2}\right]$ is given by

Production in $\left[0, t_{1}\right]$ - Demand in $[0, \mu]$ - Demand in $\left[0, t_{2}\right]$

$=\gamma \mathrm{D}_{0} \int_{0}^{t_{1}} t e^{-r t} d t-\mathrm{D}_{0} \int_{0}^{\mu} t e^{-r t} d t-\mathrm{D}_{0} \mu \int_{\mu}^{t_{2}} e^{-r t} d t$

$=\gamma \mathrm{D}_{0}\left(\frac{t_{1}^{2}}{2}-\frac{r t_{1}^{3}}{3}\right)-\mathrm{D}_{0}\left(\frac{\mu^{2}}{2}-\frac{r \mu^{3}}{3}\right)-\mathrm{D}_{0} \mu\left[\left(t_{2}-\mu\right)-r\left(\frac{t_{2}^{2}}{2}-\frac{\mu^{2}}{2}\right)\right]$

Hence the production cost over the period $\left.\left[0, t_{1}\right)\right]$ is given by

$$
\begin{aligned}
& \int_{0}^{t_{1}} K v e^{-r u} d u=\int_{0}^{t_{1}} \frac{\alpha_{1} \gamma}{R^{s-1}} e^{-r u} \mathrm{du} \\
& =\alpha_{1} \gamma D_{0}^{1-s} \int_{0}^{t_{1}} u^{1-s}(1-\mathrm{ru}) \mathrm{du} \\
& =\alpha_{1} \gamma D_{0}^{1-s}\left[\frac{t_{1}^{2-s}}{(2-s)}-\frac{r t_{1}^{1-s}}{(1-s)}\right]
\end{aligned}
$$

Shortage cost over the period $[0, \mathrm{~T}]$ is given by

$$
\begin{aligned}
& \int_{t_{2}}^{t} \theta(t) d t=-\int_{t_{2}}^{T} D_{0} \mu\left[\left\{t_{2}-t\right)-\delta\left[T-t_{2}\right]\left(t_{2}-t\right)\right] d t e^{-r t} \\
& =-D_{0} \mu\left[\left\{T t_{2}-\frac{t_{2}^{2}}{2}-\frac{T^{2}}{2}-\delta\left(\frac{3 t_{2} T^{2}}{2}-\frac{3 t_{2}^{2} T}{2}-\frac{T^{2}}{2}+\frac{t_{2}^{3}}{2}\right)-r \frac{T^{2} t_{2}}{2}+r \frac{t_{2}^{3}}{2}+r \frac{T^{3}}{3}+\delta r\left(T-t_{2}\right)\left(\frac{t_{2} T^{2}}{2}-\frac{t_{2}^{3}}{6}-\frac{T^{3}}{3}\right)\right\}\right]
\end{aligned}
$$

Lost sales cost per cycle is

$\mathrm{LS}=D_{0} \mu \int_{t_{2}}^{T}\left(1-e^{-\delta\left(T-t_{2}\right)}\right) d t$

$\mathrm{LS}=D_{0} \mu \int_{t_{2}}^{T} \delta\left(T-t_{2}\right)\left(t_{2}-t\right) d T$

Lost sales cost over the period $[0, \mathrm{~T}]$ is

$=D_{0} \mu \delta\left[\frac{3 T^{2} t_{2}}{2}-\frac{3 t_{2}^{2} T}{2}-\frac{T^{3}}{2}+\frac{t_{2}^{3}}{2}-\frac{5 r t_{2} T^{3}}{6}-\frac{r t_{2}^{4}}{6}+\frac{r T t_{2}^{3}}{6}+\frac{r T^{4}}{3}+\frac{r t_{2}^{2} T^{2}}{2}\right]$

From (39)(40),(41),(42),(43), the total average inventory cost $X$ of the system is

$$
\begin{aligned}
& \mathrm{X}=\frac{1}{t_{2}}\left[X _ { 1 } \left\{D_{0}(\gamma-1)\left[\frac{t_{1}^{3}}{6}-\frac{\alpha \beta t_{1}^{\beta+3}}{2(\beta+2)(\beta+3)}-\frac{\alpha^{2}(3 \beta+2) t_{1}^{2 \beta+3}}{2(2 \beta+2)(\beta+2)(2 \beta+3)}-\frac{r t_{1}^{4}}{8}+\frac{\alpha \beta r t_{1}^{\beta+4}}{2(\beta+2)(\beta+4)}+\frac{r \alpha^{2}(3 \beta+2) t_{1}^{2 \beta+4}}{2(2 \beta+2)(\beta+2)(2 \beta+4)}\right]+\right.\right. \\
& D_{0}\left[\frac{\gamma \mu t_{1}^{2}}{2}-\frac{\gamma t_{1}^{3}}{2}+\frac{\alpha \gamma t_{1}^{\beta+2} \mu^{2}}{\beta+2}-\frac{\alpha \gamma \beta t_{1}^{\beta+3}}{2(\beta+2)(\beta+1)}+\frac{\alpha^{2} \gamma t_{1}^{2 \beta+2} \mu}{2(2 \beta+2)}-\frac{\alpha \beta t_{1}^{\beta+3}}{2(\beta+2)(\beta+3)}-\frac{\mu^{3}}{6}+\frac{t_{1}^{3}}{6}+\frac{\alpha \beta \mu^{\beta+3}}{2(\beta+2)(\beta+3)}+\right. \\
& \frac{\alpha^{2}(3 \beta+2) \mu^{2 \beta+3}}{2(2 \beta+2)(2 \beta+3)(\beta+2)}-\frac{\alpha \gamma \mu^{\beta+1} t_{1}^{2}}{2(\beta+1)}-\frac{\alpha^{2} \gamma \mu^{\beta+1} t_{1}^{\beta+2}}{(\beta+1)(\beta+2)}-\frac{\alpha^{2}(3 \beta+2) t_{1}^{2 \beta+3}}{2(2 \beta+2)(2 \beta+3)(\beta+2)}-\frac{\alpha^{2} \gamma(\beta-2) t_{1}^{2 \beta+3}}{4(\beta+1)(\beta+2)}-\frac{r \gamma t_{1}^{2} \mu^{2}}{4}+\frac{r \gamma t_{1}^{4}}{4}- \\
& \frac{r \alpha \gamma t_{1}^{\beta+2} \mu^{2}}{2(\beta+2)}-\frac{r \gamma \alpha^{2} t_{1}^{2 \beta+2} \mu^{2}}{8(\beta+1)}+\frac{r \gamma \alpha^{2}\left(\beta^{2}-4 \beta-4\right) t_{1}^{2 \beta+4}}{8(\beta+1)(\beta+2)^{2}}+\frac{r \mu^{4}}{8}-\frac{r \alpha \beta \mu^{\beta+4}}{2(\beta+2)(\beta+4)}+\frac{r \alpha \beta t_{1}^{\beta+4}}{2(\beta+2)(\beta+4)}-\frac{r \alpha^{2}(3 \beta+2) \mu^{2 \beta+4}}{8(\beta+1)(\beta+2)^{2}}+ \\
& \left.\frac{r \alpha^{2}(3 \beta+2) t_{1}{ }^{2 \beta+4}}{8(\beta+2)^{2}(\beta+1)}+\frac{r \alpha \gamma \mu^{\beta+2} t_{1}^{2}}{2(\beta+2)}+\frac{r \alpha^{2} \gamma t_{1}^{\beta+2} \mu^{\beta+2}}{(\beta+2)^{2}}\right]+D_{0} \mu\left[\frac{t_{2}^{2}}{2}-t_{2} \mu+\frac{\mu^{2}}{2}-\frac{\alpha \mu t_{2}^{\beta+1}}{(\beta+1)}+\frac{\alpha \beta t_{2}^{\beta+2}}{(\beta+1)(\beta+2)}-\frac{\alpha \beta \mu^{\beta+2}}{(\beta+1)(\beta+2)}+\right. \\
& \frac{\alpha \mu^{\beta+1} t_{2}}{(\beta+1)}+\frac{\alpha^{2}(\beta-1) t_{2}^{2 \beta+2}}{2(2 \beta+2)(\beta+1)}-\frac{\alpha^{2} \mu t_{2}{ }^{2 \beta+1}}{2(2 \beta+1)}-\frac{\alpha^{2}(3 \beta+1) \mu^{2 \beta+2}}{2(2 \beta+1)(2 \beta+2)(\beta+1)}+\frac{\alpha^{2} \mu^{\beta+1} t_{2}{ }^{\beta+1}}{(\beta+1)^{2}}-\frac{r t_{2}^{3}}{6}+\frac{r \mu^{2} t_{2}}{2}-\frac{r \mu^{3}}{3}-\frac{\alpha \beta r t_{2}^{\beta+3}}{2(\beta+2)(\beta+3)}+ \\
& \left.\frac{r \alpha \mu^{2} t_{2}^{\beta+1}}{2(\beta+1)}+\frac{\alpha r \beta \mu^{\beta+3}}{(\beta+1)(\beta+3)}-\frac{r \alpha \mu^{\beta+2} t_{2}}{(\beta+2)}-\frac{r \alpha^{2}(\beta-2) t_{2}^{2 \beta+3}}{4(\beta+2)(2 \beta+3)}+\frac{r \alpha^{2} \mu^{2} t_{2}^{2 \beta+1}}{4(2 \beta+1)}+\frac{r \alpha^{2}(3 \beta+1) \mu^{2 \beta+3}}{2(\beta+1)(2 \beta+3)(2 \beta+1)}\right\}+X_{3}\left\{\gamma D_{0}\left(\frac{t_{1}^{2}}{2}-\frac{r t_{1}^{3}}{3}\right)-\right. \\
& \left.D_{0}\left(\frac{\mu^{2}}{2}-\frac{r \mu^{3}}{3}\right)-D_{0} \mu\left(\left(t_{2}-\mu\right)-r\left(\frac{t_{2}^{2}}{2}-\frac{\mu^{2}}{2}\right)\right)\right\}-X_{4} D_{0} \mu\left[\left\{T t_{2}-\frac{t_{2}^{2}}{2}-\frac{T^{2}}{2}-\delta\left(\frac{3 t_{2} T^{2}}{2}-\frac{3 t_{2}^{2} T}{2}-\frac{T^{3}}{2}+\frac{t_{2}^{3}}{2}\right)-\right.\right. \\
& \left.r \frac{T^{2} t_{2}}{2}+r \frac{t_{2}^{3}}{2}+r \frac{T^{3}}{3}+\delta r\left(T-t_{2}\right)\left(\frac{t_{2} T^{2}}{2}-\frac{t_{2}^{3}}{6}-\frac{T^{3}}{3}\right)\right\}+X_{5} D_{0} \mu \delta\left\{\frac{3 T^{2} t_{2}}{2}-\frac{3 t_{2}^{2} T}{2}-\frac{T^{3}}{2}+\frac{t_{2}^{3}}{2}-\frac{5 r t_{2} T^{3}}{6}-\frac{r t_{2}^{4}}{6}+\right. \\
& \left.\left.\frac{r T t_{2}^{3}}{6}+\frac{r T^{4}}{3}+\frac{r t_{2}^{2} T^{2}}{2}\right\}+\alpha_{1} \gamma D_{0}^{1-s}\left\{\frac{t_{1}^{2-s}}{(2-s)}-\frac{r t_{1}^{1-s}}{(1-s)}\right\}\right] \\
& X_{1}=X_{01}+\frac{X_{3}^{\prime}}{n^{k}}
\end{aligned}
$$


Where $X_{1}$ is continuously deceases over $n$ since $\frac{\mathrm{dx}_{1}}{\mathrm{dn}}<0, \mathrm{n}>0$

$$
\mathrm{X}_{3}=\mathrm{X}_{03}+\frac{\mathrm{X}_{3}^{\prime}}{\mathrm{n}^{\mathrm{k}}}
$$

Where $X_{3}$ is continuously deceases over $n$ since $\frac{\mathrm{dX}_{3}}{\mathrm{dn}}<0, \mathrm{n}>0$

$$
\mathrm{X}_{4}=X_{04}+\frac{X_{4}^{\prime}}{n^{k}}
$$

Where $X_{4}$ is continuously deceases over $n$ since $\frac{d X_{4}}{d n}<0, n>0$

$$
\mathrm{X}_{5}=\mathrm{X}_{05}+\frac{\mathrm{X}_{5}^{\prime}}{\mathrm{n}^{\mathrm{k}}}
$$

Where $X_{5}$ is continuously deceases over $n$ since $\frac{d X_{4}}{d n}<0, n>0$

Optimum values of $t_{1}$ and $t_{2}$ for minimum average cost are obtained as in Case 1 which gives

$X_{1}\left\{(\gamma-1) D_{o}\left(\frac{t_{1}^{2}}{2}-\frac{\alpha \beta t_{1}^{\beta+2}}{2(\beta+2)}\right)-\frac{\alpha^{2}(3 \beta+2) t_{1}^{2 \beta+2}}{2(2 \beta+2)(\beta+2)}-\frac{r t_{1}^{3}}{2}+\frac{\alpha \beta r(3 \beta+2) t_{1}^{\beta+3}}{2(\beta+2)}+\frac{r \alpha^{2}(3 \beta+2) t_{1}^{2 \beta+3}}{2(2 \beta+2)(\beta+2)}\right)+D_{o}\left(\gamma \mu t_{1}-\frac{3}{2} \gamma t_{1}^{2}+\right.$ $\alpha \gamma \mu^{2} t_{1}^{\beta+1}-\frac{\alpha \gamma \beta(\beta+3) t_{1}^{\beta+2}}{2(\beta+2)(\beta+1)}+\frac{\alpha^{2} \gamma t_{1}^{2 \beta+1} \mu}{2}-\frac{\alpha \beta t_{1}^{\beta+2}}{2(\beta+2)}+\frac{t_{1}^{2}}{2}-\frac{\alpha \gamma \mu^{\beta+1} t_{1}}{(\beta+1)}-\frac{\alpha^{2} \gamma \mu^{\beta+1} t_{1}^{\beta+1}}{(\beta+1)}-\frac{\alpha^{2}(3 \beta+2) t_{1}^{2 \beta+2}}{2(2 \beta+2)(\beta+2)}-$

$\frac{\alpha^{2} \gamma(\beta-2) t_{1}^{2 \beta+2}(2 \beta+3)}{4(\beta+1)(\beta+2)}-\frac{r \gamma t_{1} \mu^{2}}{2}+r \gamma t_{1}^{3}-\frac{r \alpha \gamma \mu^{2} t_{1}^{\beta+1}}{2}-\frac{r \gamma \alpha^{2} \mu^{2}(2 \beta+2) t_{1}^{2 \beta+1}}{8(\beta+1)}+\frac{r \gamma \alpha^{2}\left(\beta^{2}-4 \beta-4\right) t_{1}^{2 \beta+3}}{4(\beta+1)(\beta+2)}-\frac{r t_{1}^{2}}{2}+\frac{r \alpha \beta t_{1}^{\beta+3}}{2(\beta+2)}+$ $\left.\frac{r \alpha^{2}(3 \beta+2) t_{1}^{2 \beta+3}}{4(\beta+2)(\beta+1)}+\frac{r \alpha \gamma \mu^{\beta+2} t_{1}}{(\beta+2)}+\frac{r \alpha^{2} \gamma t_{1}^{\beta+1} \mu^{\beta+2}}{(\beta+2)}\right\}+X_{3} \gamma D_{o}\left(t_{1}-r t_{1}^{2}\right)+\alpha_{1} \gamma D_{o}^{1-s}\left(t_{1}^{1-s}-r t_{1}-s\right)=0$

and

$$
\begin{aligned}
& X_{1} D_{0} \mu\left(t_{2}-\mu-\alpha \mu t_{2}^{\beta}+\frac{\alpha \beta t_{2}^{\beta+1}}{(\beta+1)}+\frac{\alpha \mu^{\beta+1}}{(\beta+1)}+\frac{\alpha^{2}(\beta-1) t_{2}^{2 \beta+1}}{2(\beta+1)}-\frac{\alpha^{2} \mu t_{2}^{2 \beta}}{2}+\frac{\alpha^{2} \mu^{\beta+1} t_{2}^{\beta}}{(\beta+1)}-\frac{r t_{2}^{2}}{2}+\frac{r \mu^{2}}{2}-\frac{\alpha \beta r t_{2}^{\beta+2}}{2(\beta+2)}+\right. \\
& \left.\frac{r \alpha \mu^{2} t_{2}^{\beta}}{2}-\frac{r \alpha \mu^{\beta+2}}{(\beta+2)}-\frac{r \alpha^{2}(\beta-2) t_{2}^{2 \beta+2}}{4(\beta+2)}+\frac{r \alpha^{2} \mu^{2} t_{2}^{2 \beta}}{4}\right)-X_{3} D_{0} \mu\left(1-r t_{2}\right)-X_{4} D_{0} \mu\left\{\left(T-t_{2}\right)-\delta\left(\frac{3 T^{2}}{2}-3 t_{2} T+\right.\right. \\
& \left.\left.\frac{3^{t_{2}^{2}}}{2}\right)-\frac{r T^{2}}{2}+\frac{3}{2} r t_{2}^{2}+\frac{2}{3} \delta r t_{2}^{3}-\delta r t_{2} T^{2}+\frac{5}{6} \delta r T^{3}-\frac{1}{2} \delta r T t_{2}^{2}\right\}+X_{5} D_{0} \mu \delta\left(\frac{3}{2} T^{2}-3 t_{2} T+\frac{3}{2} t_{2}^{2}-\frac{5 r T^{3}}{6}-\frac{2}{3} r t_{2}^{3}+\right. \\
& \left.\frac{r T t_{2}^{2}}{2}+r t_{2} T^{2}\right)-X=0
\end{aligned}
$$

\section{NUMERICAL EXAMPLES}

Let us consider the inventory system with following data for case $I\left(\mu \leq t_{1} \leq t_{2}\right)$

Data for case $I\left(\mu \leq t_{1} \leq t_{2}\right)$

$\mathrm{D}_{0}=14, \mathrm{~s}=1.8, \mu=2, \alpha_{1}=2.5, \beta=0.06, \alpha=0.08, \gamma=2, \mathrm{r}=0.03, \mathrm{X}_{01}=14, \mathrm{X}_{1}{ }^{\prime}=8, \mathrm{X}_{03}=1.2, \mathrm{X}_{3}{ }^{\prime}=0.2, \mathrm{X}_{04}=12$,

$\mathrm{X}_{4}{ }^{\prime}=6, \mathrm{X}_{05}=2.9, \mathrm{X}_{5}{ }^{\prime}=0.6, \delta=0.3, \mathrm{~T}=5, \mathrm{n}=2, \mathrm{k}=1$

Output results are

$\mathrm{t}_{1}=2.056569, \mathrm{t}_{2}=3.0112976$, T.C. $=203.015$

Graphical representation of the converities of $t_{1}$ and $t_{2}$ w.r.t. T.C for case 1 . $\left(\mu \leq t_{1}\right.$ $\left.\leq t_{2}\right)$

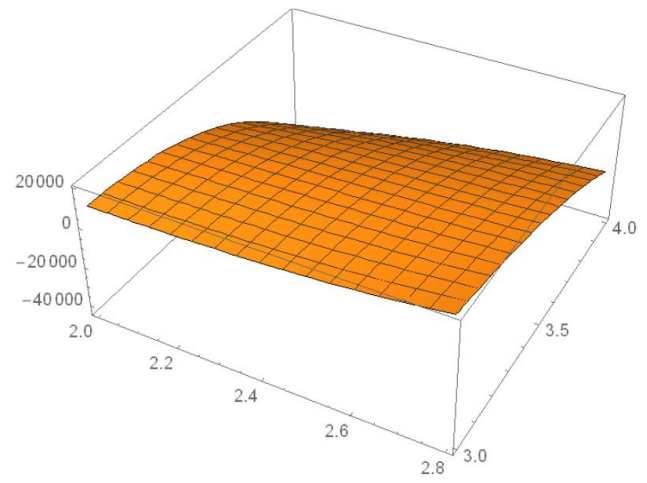

Convexity of $t_{1}$ and $t_{2}$ w.r.t T.C 
TABLE 1: SENSITIVITY ANALYSIS: THE SENSITIVITY ANALYSIS OF THE KEY PARAMETER R, $\mathrm{D}_{0}, \mathrm{~A}, \mathrm{~B}$ ARE GIVEN IN THE BELOW TABLE FOR CASE I

\begin{tabular}{|c|c|c|c|c|}
\hline PARAMETERS & & $\mathbf{t}_{1}$ & $\mathbf{t}_{2}$ & T.C. \\
\hline $\mathbf{r}$ & $\begin{array}{l}0.31 \\
0.32 \\
0.33 \\
0.34\end{array}$ & $\begin{array}{l}2.05202 \\
2.04751 \\
2.04303 \\
2.03859\end{array}$ & $\begin{array}{l}3.00235 \\
2.99348 \\
2.98468 \\
2.97595\end{array}$ & $\begin{array}{l}197.976 \\
192.977 \\
188.018 \\
183.098\end{array}$ \\
\hline $\mathbf{D}_{0}$ & $\begin{array}{l}14 \\
15 \\
16 \\
17\end{array}$ & $\begin{array}{l}2.05656 \\
2.05668 \\
2.05677 \\
2.05685\end{array}$ & $\begin{array}{l}3.01129 \\
3.01125 \\
3.01121 \\
3.01118\end{array}$ & $\begin{array}{c}203.015 \\
217.077 \\
231.16 \\
245.261\end{array}$ \\
\hline$\alpha$ & $\begin{array}{l}0.09 \\
0.10 \\
0.11 \\
0.12\end{array}$ & $\begin{array}{l}2.08359 \\
2.11048 \\
2.13719 \\
2.16367\end{array}$ & $\begin{array}{l}2.99942 \\
2.98741 \\
2.97527 \\
2.96302\end{array}$ & $\begin{array}{c}193.258 \\
183.475 \\
173.67 \\
163.85\end{array}$ \\
\hline $\boldsymbol{\beta}$ & $\begin{array}{l}0.06 \\
0.07 \\
0.08 \\
0.09\end{array}$ & $\begin{array}{l}2.05656 \\
2.05859 \\
2.06064 \\
2.06270\end{array}$ & $\begin{array}{l}3.01129 \\
3.01060 \\
3.00991 \\
3.00920\end{array}$ & $\begin{array}{c}203.015 \\
202.824 \\
202.63 \\
202.434\end{array}$ \\
\hline
\end{tabular}

The Following points are observed

1. $t_{1} \delta t_{2}$ decrease and T.C. also decreases with the increase in value of the parameter $r$

2. $t_{1} \delta$ T.C. increase while $t_{2}$ decreases with the increase in value of the parameter $D_{0}$.

3. $t_{1}$ increases while $t_{2}$ and T.C. decrease with the increase in value of the parameter $\alpha$.

4. $t_{1}$ increases while $t_{2}$ and T.C. decrease with the increase in value of the parameter $\beta$.

\section{CONCLUSION}

In this study, an EOQ model with ramp type demand rate and unit production cost under learning and inflationary environment has been developed. The quality and quantity of goods decrease in course of time due to deterioration is a natural phenomena .Hence consideration of Weibull distribution time varying deterioration function defines a significant meaning of perishable, volatile and failure of any kind of item. Shortages are allowed and partially backlogged. The two considered phenomena viz., learning and inflation play an important role in realistic scenario. A mathematical model has been found to determine the optimal ordering policy cost which minimizes the present worth of total optimal cost. Thus the model highlighted the results with numerical examples.

Equation (24) and (25) are non- linear equation in $\mathrm{t}_{1}$ and $\mathrm{t}_{2}$. These simultaneous non-linear equations can be solved for suitable choice of the parameters $\mathrm{X}_{1}, \mathrm{X}_{3}, \mathrm{X}_{4}, \mathrm{X}_{5}, n, k, \alpha, \beta, r, \gamma, \mu, \delta, D_{0}, \alpha_{1}$ and $\mathrm{s}(\neq 2)$. If $t_{1}^{*}$ and $t_{2}^{*}$ are the solution of (24) and (25) for Case $\mathrm{I}$, the corresponding minimum cost $X^{*}\left(\mathrm{t}_{1}, \mathrm{t}_{2}\right)$ can be obtained from (23). It is very difficult to show analytically whether the cost function $X\left(t_{1}, t_{2}\right)$ is convex. That is why, $X\left(t_{1}\right.$, $t_{2}$ ) may not be global minimum. If $X\left(t_{1}, t_{2}\right)$ is not convex, then $X\left(t_{1}, t_{2}\right)$ will be local minimum.

Similarly, solution of equations (45) and (46) for Case II can be obtained corresponding minimum cost X ( $\mathrm{t}_{1}$, $\mathrm{t}_{2}$ ) can be obtained from (44).

\section{REFERENCES}

[1] Abad, P.L. (1996), Optimal pricing and lot sizing under conditions of perishability and partial backlogging, Management Science, 42 (8) , 1093-1104.

[2] Buzzacott, J.A. (1975), Economic order quantities with inflation, Operation Research Quarterly, 26(3), 553-558.

[3] Covert, R.P., Philip, G.C. (1973), An EOQ model for items with Weibull distribution deterioration. AIIE Transactions, 5(4), 323-326. 
[4] Deng, P.S., Lin, R.H.J., Chu, P.(2007), A note on the inventory models for deteriorating items with ramp type demand rate, European Journal of Operational Research ,178(1),112-120.

[5] Dye, C.Y., Ouyang, L.Y. and Hsieh, T.P. (2007), Deterministic inventory model for deteriorating items with capacity constraint and time proportional backlogging rate, European Journal of Operational Research, 178(3), 789807.

[6] Ghare, P. M. and Schrader, G. F., (1963) A model for exponentially decaying inventories, Journal of Industrial Engineering, 14, 238-243.

[7] Giri, B.C., Jalan, A.K. and Chaudhuri, K.S.(2003), Economic order quantity model with Weibull deterioration distribution shortage and ramp type demand, International Journal of Systems Science, 34 (4), 237-243.

[8] Hill, R.M. (1995), Inventory model for increasing demand followed by level demand, The Journal of the operational Research Society, 46,1250-1259.

[9] Jayshree and Jain S. (2016), An EOQ model dealing with Weibull deterioration With shortages, ramp type demand rate and unit production cost incorporating the effect of inflation." Sixth International Conference on Computational Intelligence \& Information Technology- CIIT-2016, McGraw-Hill Conference Proceeding, 150-163.

[10] Jayshree and Singh S.R. (2016), "An inventory model for decaying items with ramp type demand under learning effect," Seventh International Conference on Advances in Computing, Control, and Telecommunication Technologies - ACT 2016 pp-276-283.

[11] Kumar, N., Singh, S.R., and Kumari, R. (2013) Learning effect on an inventory model with two-level storage and partial backlogging under inflation. International Journal of Services and Operations Management. Vol. 16, Issue 1, pp. 105-122

[12] Mandal B. and Pal A.K., (1998)"Order level inventory system with ramp type demand rate for deteriorating items," Journal of Interdisciplinary Mathematics, vol.1, no. 1,pp. 49-66.

[13] Manna, P., Manna, S.K., Giri, B.C. (2016), An economic order quantity model with ramp type demand rate, constant deterioration rate and unit production cost, Yugoslav Journal of Operations Research,

[14] Manna, S.K and Chaudhuri, K.S.(2006), An model with ramp type demand rate, time dependent deterioration rate, unit production cost and shortages, European Journal of Operational Research, 171 (2), 557-566.

[15] Misra, R.B. (1975). Optimum production lot-size model for a system with deteriorating inventory. International Journal of Production Research. 13 (5), 495-505.

[16] Singh C, Singh S.R. (2011), "Imperfect production process with exponential demand rate, Weibull deterioration under inflation", International Journal of Operational Research 12 (4), 430-445

[17] Singh S, Jain S., Pareek S. (2013), "An imperfect quality items with learning and inflation under two limited storage Capacity,” International Journal of Industrial Engineering Computations 4(4), 479-490

[18] Singh S.R., Kumar N., Kumari R. (2009), "Two warehouse inventory model for deteriorating items with shortages under inflation and time value of money", International Journal of Computational and Applied Mathematics 4 (1), 83-94.

[19] Singh, S.R. and Singh, T.J. (2007), An EOQ inventory model with weibull distribution deterioration, ramp type demand and partial backlogging rate, Indian Journal of Mathematics and Mathematical Science ,3(2),127-137.

[20] Singh, T.J., Singh, S.R. and Singh, C. (2008), Perishable inventory model with quadratic demand, partial backlogging and permissible delay in payments, International Review of Pure and Applied Mathematics, 53-66.

[21] Skouri, K., Konstantaras, I., Manna, S. K. and Chaudhuri, K. S. (2011) Inventory models with ramp type demand rate, partial backlogging and Weibull deterioration rate, European Journal of Operational Research, 192(1) 79-92.

[22] Wu, K. S., "An EOQ inventory model for items with Weibull distribution deterioration, ramp type demand rate and partial backlogging”, Production Planning and Control, 12 (2001) 787-793

[23] Wu, K.S., Ouyang, L.Y. and Yang, C.T. (2006), An optimal replenishment policy for non-instantaneous deteriorating items with stock dependent demand and partial backlogging, International Journal of Production Economics, 101, 369-384.

[24] Yadav, D., Singh, S.R., and Kumari, R. (2011), Optimization policy of inventory model under the effects of learning and imprecise demand rate, International Journal of Inventory Control and Management, 1(1), 49-69.

[25] Yang G. K., Lin R., Lin J., Hung K,-C, , Chu P, , and Chouhuang W., "Note an inventory models with Weibull distribution deterioration," Production planning \& Control, vol. 22, no. 4, pp, 437-444, 2011.

[26] Yang, H.L., (2012). Two -warehouse partial backlogging inventory models with three parameter Weibull distribution deterioration under inflation International Journal of Production Economics 138 (1), 107-116.

[27] Yang, H.L., Teng, J.T. and Chern, M.S.(2001),Deterministic inventory lot -size models under inflation with shortages and deterioration for fluctuating demand, Naval Research Logistics, 48, 144-158. 